\title{
Vaccination rates and attitudes to vaccination in a tertiary hospital RA clinic cohort
}

\author{
Lee $\mathrm{JH}^{1,2}$, Taur $\mathrm{S}^{1,2}$, Eades $\mathrm{L}^{2}$, Nalpanitis $\mathrm{T}^{1}$, Antony $\mathrm{A}^{1}$, Kemp $\mathrm{S}^{1}$, Morton $\mathrm{S}^{1}$, Dendle $\mathrm{C}^{1,2}$ and Leech $\mathrm{M}^{1,2}$ \\ ${ }^{1}$ Department of Rheumatology, Monash Health, Clayton, Victoria, Australia \\ ${ }^{2}$ Faculty of Medicine, Nursing and Health Sciences, Monash University, Victoria, Australia
}

\begin{abstract}
Background: The increasing use of immunosuppressive drugs and reports of infection rates in rheumatoid arthritis (RA) registries highlights the need to understand rates of common vaccinations, influenza and pneumococcal, and attitudes to vaccination in Australian RA cohorts.

Aims: To investigate influenza and pneumococcal vaccination rates and attitudes to vaccination in a specialist RA clinic and to examine the infection risk in unvaccinated patients

Methods: A cross sectional study of 193 consecutive RA clinic patients was performed using a survey of influenza and pneumococcal vaccination status, and reasons for non-vaccination were documented. Infection risk in non- or never-vaccinated patients was calculated using the Rheumatoid Arthritis Observation of Biologic Therapy (RABBIT) risk score.

Results: RA cohort demographics were representative of a standard RA population. $70 \%$ of patients were seropositive and $29 \%$ were current smokers. $83 \%$ of patients were on a non-biological disease modifying anti-rheumatic drug, most commonly methotrexate (67\%) and hydroxychloroquine (33\%). 38\% of patients were on biological disease modifying anti-rheumatic drugs (bDMARDs), predominantly anti-TNF alpha inhibitors. $43 \%$ of patients were currently taking steroids. The mean DAS28 in this cohort was 3.3, suggesting moderately active disease. 34\% of patients were unvaccinated, of which half (51\%) had never been vaccinated. In the never vaccinated group and the not-currently vaccinated group, the commonest reason for non-vaccination was 'worries about the safety of the vaccine, including side effects'. The mean age in the non-vaccinated group was 48 , the mean steroid dose was $1.96 \mathrm{mg}$, average numbers of DMARDs in this group was 2.9 concomitant biologic therapy was prescribed in $46 \%$ of patients. The risk of infection was between $1.4-3.5 \% /$ year.

Conclusion/impact: This study highlights incomplete influenza and pneumococcal vaccination in an at-risk immunosuppressed Australian RA cohort. Concern regarding vaccine safety was the primary reason for non- vaccination. This highlights the need to tailor information strategies to particular sub groups within the cohort. The uptake of emerging vaccines is likely to be influenced by prevailing attitudes to vaccination and a patient centred approach which quantifies and explains risk -benefit for individual patients may be required to translate the therapeutic impact to whole cohorts of immunosuppressed patients.
\end{abstract}

\section{Introduction}

Rheumatoid arthritis (RA) patients are at twice the risk of developing infections compared to individuals of similar characteristics who do not have RA [1]. This increased risk of infections is likely multifactorial. Firstly, studies have shown that rheumatoid arthritis causes immune system dysregulation, including $\mathrm{T}$ cell abnormalities, which likely compromises the immune system's ability to respond to novel antigenic stimuli [2]. Secondly, co-morbidities, such as diabetes mellitus and chronic lung disease, are common in the RA cohort, and have been shown to be a strong predictor of infection in this cohort of patients [3]. Thirdly, immunosuppressive drugs used to treat RA are also largely responsible for the heightened risk of infection in RA patients. Indeed, with more and more new biological therapies, the use of immunosuppressive therapies is increasing, placing RA patients at greater risk of infection than before.

These risk factors highlight the need for and importance of vaccinations in this group of patients to prevent infections, and their associated morbidity and mortality. Current Australian, European League Against Rheumatism (EULAR) and American College of Rheumatology (ACR) guidelines all recommend annual influenza vaccination, and pneumococcal vaccine every 5 years for RA patients $[4,5]$. However, despite these guidelines, the infection rates in RA registries and data from international RA cohorts suggest that there may be suboptimal uptake of these recommendations in actual clinical practice. In a large cohort of RA patients enrolled across 17 countries, Hmamouchi et al found that overall vaccination coverage was low (17.2\% for pneumococcal vaccination and $25.3 \%$ for influenza vaccinations) with huge disparities between countries [6]. Costello et al found in a retrospective cohort study of RA patients in the UK that one in five RA patients received no influenza vaccinations, and only half of their RA patients received the pneumococcal vaccine [7]. Interestingly, there is relatively less data on uptake of vaccinations in Australian cohorts of RA patients.

With this in mind, the aim of our study was to firstly, investigate current vaccination rates and attitudes to vaccination for vaccinepreventable infection, namely influenza and pneumococcal infections, in an Australia RA patient cohort, examine the infection risk in

Correspondence to: Jia Hui Lee, Faculty of Medicine, Nursing and Health Sciences, Monash University, Australia Tel: +61 39905 8134; E-mail: lee_jiahui@hotmail.com

Received: November 18, 2016; Accepted: December 23, 2016; Published: December 27, 2016 
unvaccinated RA patients $[1,2]$.

\section{Methods}

We performed a cross sectional study of 193 consecutive public RA specialist clinic patients in an Australian tertiary hospital.

A written patient survey was developed by the authors in collaboration with consultant rheumatologists at our large tertiary centre. Patients indicated their consent to participate on the survey form. Data collected included vaccination history for influenza and pneumococcus, reasons for vaccination or non-vaccination, and patient's perception of their risk of acquiring an infection.

The demographics of this RA cohort were recorded including gender, age, seropositivity, smoking status, Disease Activity Score (DAS28), and their use of steroids, or a biologic agent. In the unvaccinated (non- or never-vaccinated) cohort, the mean steroid dose and average number of disease modifying anti-rheumatic drugs (DMARDs) were also recorded.

Infection risk in the next 12 months in the non- or never-vaccinated patients was then determined using the Rheumatoid Arthritis Observation of Biologic Therapy (RABBIT) risk score, an instrument which determines the risk of serious infection in rheumatoid arthritis patients based on clinical and treatment information [8].

\section{Results}

193 RA patients participated in the written survey. The overall RA cohort demographics were representative of a standard RA population (see Table 1). The mean age of patients recruited was 60 , and nearly three-quarters of patients were female. $70 \%$ of patients were seropositive and $29 \%$ were current smokers. $83 \%$ of patients were on a non-biological disease modifying anti-rheumatic drug, most commonly methotrexate (67\%) and hydroxychloroquine $(33 \%)$. $38 \%$ of patients were on biological disease modifying anti-rheumatic drugs (bDMARDs), predominantly anti-TNF alpha inhibitors. $43 \%$ of patients were currently taking steroids. The mean DAS28 in this cohort was 3.3 , suggesting moderately active disease.

$34 \%$ of patients were unvaccinated for influenza, of which half (51\%) had never been vaccinated for influenza. In terms of uptake of pneumococcal vaccines, our study found that $65 \%$ of patients were unvaccinated for pneumococcal, of which $89 \%$ had never been vaccinated for pneumococcal, whilst $11 \%$ had not received the vaccine in the last 5 years. In the never vaccinated group and the not-currently

Table 1. RACohort Demographics.

\begin{tabular}{|l|l|}
\hline Mean age & $\mathbf{6 0}$ years \\
\hline \% Female & $73 \%$ \\
\hline Seropositivepatients & $70 \%$ \\
\hline Current smoker & $29 \%$ \\
\hline $\begin{array}{l}\text { DiseaseModifyingAnti-RheumaticDrugs (DMARD) } \\
\text { use }\end{array}$ & $\begin{array}{l}83 \% \text { (total) } \\
- \text { Methotrexate: } 67 \% \\
- \text { Hydroxychloroquine: } 33 \% \\
- \text { Sulfasalazine21\% } \\
- \text { Leflunomide } 13 \%\end{array}$ \\
\hline $\begin{array}{l}\text { BiologicalDiseaseModifyingAnti- Rheumatic Drugs } \\
\text { (bDMARD) use }\end{array}$ & $38 \%$ \\
\hline Prednisolone use & $43 \%$ \\
\hline Mean DAS28 score (disease activity) & 3.3 (moderate activity) \\
\hline
\end{tabular}

vaccinated group, the commonest reason for non-vaccination was 'worried about the safety of the vaccine, including side effects'.

In the non-vaccinated group, the mean age was 48 . The mean steroid dose in this group was $1.96 \mathrm{mg}$, the average numbers of DMARDs in this group was 2.2 , concomitant biologic therapy was prescribed in $46 \%$ of patients. Using the RABBIT risk score (8), the risk of infection in this group was between 1.4-3.5\%/year.

\section{Discussion}

To our knowledge, this is the first study that has investigated vaccination rates in a large Australian RA cohort. This study highlights incomplete vaccination in an at-risk immunosuppressed Australian RA cohort. This is consistent with results from other studies, which show that immunosuppressed RA cohorts worldwide are under-vaccinated, despite international guidelines [6].

Our study shows that $34 \%$ of RA patients were unvaccinated for influenza, of which around half had never been vaccinated from influenza before. Additionally, $65 \%$ of patients were unvaccinated for pneumococcal, of which $89 \%$ had never been vaccinated for pneumococcal. Given the increased susceptibility to infection amongst RA patients, and the increasingly widespread use of new biological therapies, this sub-optimal uptake of vaccinations is concerning. Indeed, the use of effective biological therapies in RA must be balanced with the serious risk of infection. The morbidity and mortality resulting from serious infection can be just as significant as that resulting from RA itself. Thus, the management of RA should encompass vaccination, in order to optimally treat patients with immunosuppressive therapy, whilst mitigating the risk of infection that this therapy poses.

Perhaps more concerning are the reasons for non-vaccination amongst RA patients. In our study, the primary reason for nonvaccination amongst RA patients was safety about the vaccine, including side effects. Studies have shown that influenza and pneumococcal vaccinations are safe in patients with RA, and are effective in producing an antibody response even if the patient is on immunosuppressive medication [7]. Thus, our study results highlight that RA patients may not be adequately educated regarding the therapeutic benefit of vaccinations in preventing infections with serious consequences, which outweighs the comparatively small risk of vaccine side-effects. This emphasises the need to employ targeted information-education strategies to change specific attitudes within the cohort. A visual risk tool which balances estimated personalized moderate individual risk of infection with low risks of vaccine side-effects may be useful in shared decision making and promote vaccine uptake. This is in development as an intervention for the next phase of this work.

Additionally, the second most common reason for not vaccinating in the group of RA patients who have never received the influenza vaccination was 'my doctor didn't mention it' and 'I don't need the vaccine as I don't get the flu'. The absence of a recommendation for vaccination from a treating physician has been shown to be one of the main reasons for patients to be unvaccinated in many studies worldwide $[9,10]$. However, an Irish study found that most of their rheumatologists surveyed neither recommended nor recorded vaccination history in their clinical notes, and felt that the onus should be on primary care physicians to improve vaccine compliance [11].

Contrary to this, we believe that the responsibility to educate and encourage vaccination in RA patients lies with both the rheumatologist initiating treatment of RA with immunosuppressive therapy, as well 
as the primary care physician who is often the first point of access for patients to receive vaccines. Indeed, rheumatologists who have been reminded to address vaccination in their consultations with patients have been shown to have a significant increase in the rate of patients who were up-to-date with vaccinations [12].

Ultimately, the utility of vaccinations in mitigating against infection risk is only as good as their uptake in at risk populations, such as RA patients. The increasing availability of new vaccines, including herpes zoster, cannot translate to benefit in a population that is under vaccinated or has unexplored attitudes to vaccination. Improving and sustaining vaccination uptake for existing vaccines is a first critical step. Identifying specific reasons for non-vaccination in an at-risk group may better inform an intervention which individualises an intervention or risk communication tool.

\section{Conclusion}

This study highlights incomplete vaccination in an at-risk immunosuppressed Australian RA cohort. Concern regarding vaccine safety was the primary reason for non-vaccination. This highlights the need to tailor information strategies to particular sub groups within the cohort. The uptake of emerging vaccines is likely to be influenced by prevailing attitudes to vaccination and a patient centred approach which quantifies and explains risk -benefit for individual patients may be required to translate the therapeutic impact to whole cohorts of immunosuppressed patients.

\section{References}

1. Doran MF, Crowson CS, Pond GR, O'Fallon WM, Gabriel SE (2002) Frequency of infection in patients with rheumatoid arthritis compared with controls: a populationbased study. Arthritis Rheum 46: 2287-2293. [Crossref]
2. Koetz K, Bryl E, Spickschen K, O'Fallon WM, Goronzy JJ, et al. (2000) T cell homeostasis in patients with rheumatoid arthritis. Proc Natl Acad Sci USA 97: 92039208. [Crossref]

3. Doran MF, Crowson CS, Pond GR, O'Fallon WM, Gabriel SE (2002) Predictors of infection in rheumatoid arthritis. Arthritis Rheum 46: 2294-2300. [Crossref]

4. Singh JA, Furst ED, Bharat A, Curtis JR, Kavanaugh AF, et al., (2012) Update of the 2008 American College of Rheumatology Recommendations for the use of diseasemodifying antirheumatic drugs and biologic agents in the treatment of rheumatoid arthritis. Arthritis Care Res 64: 625-639.

5. van Assen S, Agmon-Levin N, Elkayam O, Cervera R, Doran MF, et al., (2011) EULAR recommendations for vaccination in adult patients with autoimmune inflammatory rheumatic diseases. Ann Rheum Dis 70: 414-422. [Crossref]

6. Hmamouchi I, Winthrop K, Launay O, Dougados M (2015) Low rate of influenza and pneumococcal vaccine coverage in rheumatoid arthritis: data from the international COMORA cohort. Vaccine 33: 1446-1452. [Crossref]

7. Costello R, Winthrop KL, Pye SR, Brown B, Dixon WG (2016) Influenza and Pneumococcal Vaccination Uptake in Patients with Rheumatoid Arthritis Treated with Immunosuppressive Therapy in the UK: A Retrospective Cohort Study Using Data from the Clinical Practice Research Datalink. PLoS One. 11(4): e0153848. [Crossref]

8. Zink A, Manger B, Kaufmann J, Eisterhues C, Krause A, et al., (2014) Evaluation of the RABBIT Risk Score for serious infections. Ann Rheum Dis 73: 1673-1676. [Crossref]

9. Hua C, Morel J, Ardouin E, Ricard E, Foret J, et al., (2015) Reasons for non-vaccination in French rheumatoid arthritis and spondyloarthritis patients. Rheumatology (Oxford) 54: 748-750. [Crossref]

10. Koutsogeorgopoulou L, Antoniadis C, Vassilopoulos D, Kassimos D (2009) Preventive influenza vaccination for patients with rheumatoid arthritis. A need for an international campaign. Clin Rheumatol 28: 103-104. [Crossref]

11. McCarthy EM, Azeez MA, Fitzpatrick FM, Donnelly S (2012) Knowledge, attitudes, and clinical practice of rheumatologists in vaccination of the at-risk rheumatology patient population. J Clin Rheumatol 18: 237-241. [Crossref]

12. Desai SP, Lu B, Szent-Gyorgyi LE, Bogdanova AA, Turchin A, et al., (2013) Increasing pneumococcal vaccination for immunosuppressed patients: a cluster quality improvement trial. Arthritis Rheum 65: 39-47. [Crossref]

Copyright: (C2016 Lee JH. This is an open-access article distributed under the terms of the Creative Commons Attribution License, which permits unrestricted use, distribution, and reproduction in any medium, provided the original author and source are credited. 